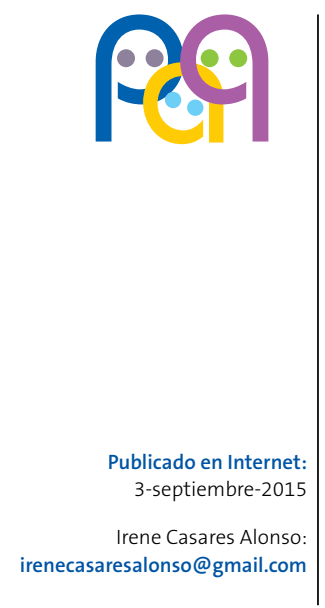

Palabras clave:

- Uso off-label

- Antiasmáticos

- Estudios de utilización

de medicamentos

- Atención Primaria

- Niños

- Estudios

retrospectivos

\title{
Prescripción off-label de antiasmáticos en Atención Primaria
}

\author{
I. Casares Alonso ${ }^{a}$, A. Cano Garcinuño ${ }^{b}$, A. Blanco Quirós ${ }^{c}$, I. Pérez García ${ }^{d}$

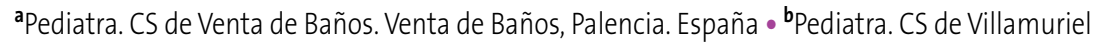

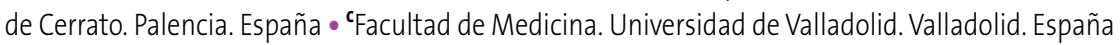 \\ - dPediatra. CS Jardinillos. Palencia. España.
}

Introducción y objetivos: se denomina prescripción off-label a la realizada fuera de las condiciones autorizadas en la ficha técnica de un medicamento. En Atención Primaria se publican cifras globales de prescripción off-label en diferentes países del 3-67\%. El objetivo del estudio ha sido conocer la intensidad de este tipo de prescripción en el caso de los antiasmáticos en un periodo reciente, en CastillaLeón (España).

Métodos: se analizaron las prescripciones correspondientes al subgrupo terapéutico R03 (medicamentos para enfermedades obstructivas respiratorias) realizadas a menores de 14 años entre 2005-2010 en el sistema público de salud autonómico. La información sobre el uso off-label se presenta en número de prescripciones consumidas de antiasmáticos a dosis y/o edades no recomendadas en su ficha técnica.

Resultados: se realizaron 394876 prescripciones de antiasmáticos a una población expuesta de 1580229 personas/año. Los menores de cuatro años fueron los que más antiasmáticos recibieron. La máxima prescripción de broncodilatadores ocurrió en menores de dos años, la de montelukast y corticoides inhalados monofármaco al año de edad. El $46 \%$ de las prescripciones de salbutamol sistémico y el $15,9 \%$ de las de la terapia de mantenimiento se prescribieron off-label.

Conclusiones: el estudio muestra un uso importante de antiasmáticos a edades muy tempranas en las que es difícil hacer el diagnóstico de asma y un consumo notable de fármacos controladores a dosis y/o edades no autorizadas. La prescripción off-label no presupone una prescripción inadecuada, pero debería tener un beneficio real para el paciente y estar apoyada por avales científicos que justifiquen su uso.

\section{Off-label prescription of anti-asthmatic agents in Primary Care}

Introduction and objectives: off-label prescribing is when performed outside of the official information of the product. Overall figures of off-label prescribing in primary care in different countries are 3-67\%. The aim of the study was to determine the intensity of this type of prescription in anti-asthmatic agents in a recent period of time, in Castilla y León.

Methods: the requirements for the therapeutic subgroup R03 (anti-asthmatic agents) prescribed in children less than 14 years of age in the Castilla-León health service from 2005 to 2010 were analyzed. Information on off-label prescribing is shown in number of anti-asthmatic prescriptions consumed at doses and/or at ages not recommended in its technical specifications.

Results: a total of 394876 prescriptions for anti-asthmatic agents were performed to an exposed population of 1580229 person-years. Children under four years received the highest levels of antiasthmatic agents. The maximum prescription of bronchodilators occurred in children under 2 years, and of montelukast and single inhaled corticosteroids at one year of age. $46 \%$ of prescriptions of systemic salbutamol and $15.9 \%$ of maintenance therapy prescriptions were made in an off-label manner.

Key words:

- Off-label use

- Anti-asthmatic agents

- Drug utilization

studies

- Primary Care

- Children

Conclusions: the study shows an important and very early use of anti-asthmatic agents at ages where it is difficult to make a diagnosis of asthma and also a remarkable consumption of controller therapy at not authorized dose and/or ages. Off-label prescription do not presuppose inappropriate prescription but it should have a real benefit to the patient and be supported by scientific guarantees to justify its use.

- Retrospective studies 


\section{INTRODUCCIÓN}

La utilización de un medicamento comercializado en condiciones no autorizadas en su ficha técnica se conoce en la literatura científica anglosajona como uso off-label. La ficha técnica es un documento requerido por las autoridades sanitarias previo a la autorización de un medicamento. Contiene la información que los profesionales sanitarios necesitan conocer para utilizar de forma segura y eficaz el fármaco. Estas condiciones de autorización pueden variar para un medicamento concreto comercializado por diferentes laboratorios en el mismo país, y también varían para el mismo medicamento en diferentes países.

Los criterios más referenciados de uso de fármacos al margen de lo aprobado en su ficha técnica incluyen su utilización en indicaciones, edades, dosis, vías de administración o en formatos farmacéuticos no autorizados ${ }^{1,2}$.

El porcentaje de prescripciones de este tipo varía en los distintos estudios en función del grupo de medicamentos analizados, del lugar en que se realiza la prescripción (Atención Especializada o Primaria), y de otros factores. Es más frecuente en salas hospitalarias, especialmente neonatales, de cuidados intensivos y quirúrgicos ${ }^{3-5}(11-80 \%$ de prescripciones). Para enfermedades alérgicas y/o respiratorias se sitúa entre el 3 y el $56 \%$ de las prescripciones y alcanza hasta el $78 \%$ de pacientes ${ }^{6-8}$. En Atención Primaria las cifras globales de prescripción off-label notificadas oscilan entre el 3 y el $67 \%$ en diferentes países ${ }^{6,9-12}$. En España no existen muchos estudios al respecto, aunque en Atención Primaria (AP), que es donde se realizan la mayor parte de las recetas, el $27-50 \%$ de las prescripciones y entre el $34-68 \%$ de niños reciben medicamentos off-label ${ }^{13-15}$. Además, hasta un $51 \%$ de pediatras reconocen haber prescrito alguna vez una medicación en estas condiciones ${ }^{16}$. A nivel mundial, los niños más pequeños son los que las reciben más frecuentemente $e^{6-7,10,12,17,18}$ y los antiasmáticos son uno de los grupos de medicamentos más habitualmente utilizados al margen de la ficha técnica ${ }^{10-12,17,19}$
El motivo de que sea tan frecuente este tipo de prescripción en la infancia es la escasez de ensayos clínicos realizados en niños a pesar de las medidas llevadas a cabo por la Food and Drug Administration (FDA) ${ }^{20,21}$ y la Agencia Europea del Medicamento (EMEA) ${ }^{22}$. Esto ha provocado que existan pocos datos sobre eficacia y seguridad en niños para muchas medicinas ${ }^{23-25}$. De hecho, existe información técnica pediátrica en el etiquetado de menos del $50 \%$ de los fármacos ${ }^{26}$.

En España el Real Decreto 1015/2009, de 19 de junio, que regula la disponibilidad de medicamentos en situaciones especiales, contempla que "este tipo de prescripción tendrá carácter excepcional, se limitará a situaciones que carezcan de alternativas terapéuticas autorizadas para un determinado paciente, y respetará las restricciones que se hayan establecido ligadas a la prescripción y/o dispensación del medicamento y el protocolo terapéutico asistencial del centro sanitario. El médico prescriptor justificará en la historia clínica la necesidad del uso del medicamento e informará al paciente de los beneficios y los riesgos potenciales, obteniendo su consentimiento" 27 .

Aunque la prescripción realizada al margen de su autorización no siempre es inadecuada, sí parece que presenta con más frecuencia problemas de reacciones adversas, que a veces son graves ${ }^{4,28-32}$. El objetivo del estudio ha sido conocer la intensidad de este tipo de prescripción en el caso de los antiasmáticos en la comunidad autónoma de Castilla y León (España), en niños de 0-14 años, en el periodo 2005-2010.

\section{POBLACIÓN Y MÉTODOS}

Se trata de un estudio observacional descriptivo retrospectivo, que analiza las prescripciones correspondientes al subgrupo terapéutico R03 (medicamentos para enfermedades obstructivas respiratorias) de la Anatomical Therapeutic Chemical Classification (ATC) de la Organización Mundial de la Salud.

Los datos de consumo farmacéutico proceden del Sistema de Información de Farmacia de Castilla y 
León (CONCYLIA), que no tiene conexión con la historia clínica informatizada del paciente. Son datos de recetas oficiales del Sistema Nacional de Salud de Castilla y León (SACYL), realizadas en AP entre el 1 de enero de 2005 y el 31 de diciembre de 2010 y dispensadas en oficinas de farmacia.

Se analizaron las prescripciones indicadas a menores de 14 años y en concreto: el principio activo, la edad del paciente y el contenido y tipo de presentación. Los datos sobre prescripción off-label se presentan en número de prescripciones de antiasmáticos realizadas a edades y/o dosis no recomendadas en su ficha técnica. Además se ha considerado off-label el uso de corticoides inhalados a dosis superiores a las denominadas como "altas" por las principales guías de asma ${ }^{33-36}$. Estas cifras son también superiores a las aprobadas en ficha técnica (Tablas 1 y 2). Las fichas técnicas se han consultado en página web de la Agencia Española de Medicamentos y Productos Sanitarios (AEMPS), agencia que en España evalúa y autoriza la comercialización de los medicamentos (www.aemps.gob.es/cima/ fichasTecnicas.do?metodo=detalleForm).

Además, para cuantificar el consumo por edad se ha utilizado la dosis diaria prescrita (PDD, prescribed daily dose), que es la dosis media diaria prescrita de un fármaco determinado, en su indicación principal. Las PDD se han calculado teniendo en cuenta las dosis por edad o peso que recomiendan las fichas técnicas de los medicamentos estudiados y las principales guías de asma en la infancia. Se estimaron PDD para 26 principios activos con 59 presentaciones farmacéuticas diferentes. Para medir la intensidad del consumo por edad se ha utilizado la dosis diaria prescrita por mil habitantes y día (PDHD) que representa el promedio de dosis diarias prescritas cada día a 1000 personas expuestas. Más información sobre la metodología se expone en otro trabajo reciente ${ }^{37}$.

La población expuesta son los menores de 14 años con tarjeta sanitaria individual en Castilla-León entre los años 2005 y 2010. Los datos de población fueron facilitados por la Dirección Técnica de Atención Primaria de la Gerencia Regional de Salud de Castilla y León, que da cobertura al 96\% de la población. Los datos de identificación del paciente, del médico prescriptor y de la marca comercial empleada no fueron recogidos, para mantener el anonimato.

Para el análisis se empleó SPSS ${ }^{\oplus}$ v 5 y programación de hojas Excel ${ }^{\oplus}$.

\section{RESULTADOS}

Durante el periodo de estudio se realizaron 394876 prescripciones de antiasmáticos a una población expuesta de 1580229 personas/año. Los broncodilatadores fueron los antiasmáticos más consumidos (7,5 PDHD). De la terapia de mantenimiento, los antagonistas de leucotrienos fueron el subgrupo más prescrito (ALT: 5,2 PDHD), seguidos de los Cl monofármaco ( $\mathrm{Cl}: 4,7 \mathrm{PDHD}$ ) y de los $\mathrm{Cl}$ asociados a

\begin{tabular}{|l|c|l|}
\hline \multicolumn{1}{|c|}{ Tabla 1. Antiasmáticos con restricciones de edad en su ficha técnica (AEMPS) activo } & Código ATC & \multicolumn{1}{c|}{ Edad mínima autorizada } \\
\hline Salbutamol sistémico & RO3CC02 & 2 años \\
\hline Salmeterol & RO3AC12 & 4 años \\
\hline Formoterol & RO3AC13 & 5 años \\
\hline Budesonida inhalada & RO3BA02 & 2 años \\
\hline Fluticasona propionato inhalada & RO3BA05 & 1 año \\
\hline Salmeterol en asociación & RO3AK06 & 4 años \\
\hline Formoterol en asociación & RO3AK07 & 6 años \\
\hline Motelukast & RO3DC03 & 6 meses-5 años: 4 mg/día \\
& & $6-14$ años: $5 \mathrm{mg} /$ día \\
\hline
\end{tabular}

AEMPS: Agencia Española de Medicamentos y Productos Sanitarios; ATC: Anatomical Therapeutic Chemical Classification. 


\begin{tabular}{|c|c|c|c|c|}
\hline $\begin{array}{l}\text { Corticoides inhalados } \\
\text { (solos o en asociación) }\end{array}$ & $\begin{array}{c}\text { NAEPP }{ }^{47} \\
\text { (dosis altas) }\end{array}$ & $\begin{array}{l}\text { Gina } 2014^{48} \\
\text { (dosis altas) }\end{array}$ & $\begin{array}{l}\text { Consenso español }{ }^{49} \\
\text { (dosis altas) }^{*}\end{array}$ & Dosis "muy altas" CyL \\
\hline $\begin{array}{l}\text { Budesonida/ } \\
\text { beclometasona HFA } \\
\text { inhaladas }\end{array}$ & $\begin{array}{l}\text { 0-4 años: NA } \\
\text { 5-11 años: } \\
>800 \mathrm{Bu} />320 \mathrm{Be}\end{array}$ & $\begin{array}{l}\text { 6-11 años: }>400 \mathrm{Bu} />200 \mathrm{Be} \\
\geq 12 \text { años: }>800 \mathrm{Bu} />400 \mathrm{Be}\end{array}$ & $>400 \mathrm{Bu}$ & $<14$ años: $>800$ \\
\hline $\begin{array}{l}\text { Fluticasona propionato } \\
\text { inhalada }\end{array}$ & 0-11 años: >352 & $\begin{array}{l}\text { 6-11 años: >400DPI/>500HFA } \\
\geq 12 \text { años: >500 DPI y HFA }\end{array}$ & $>250$ & $<14$ años: $\geq 500$ \\
\hline
\end{tabular}

Be: bleclometasona HFA; Bu: budesonida; CyL: Castilla y León; HFA: hidrofluoroalcano; NA: no aprobado. ${ }^{*}$ Niños de menos de $40 \mathrm{~kg}$ de peso.

broncodilatadores de acción larga (LABA-CI: 2,2 PDHD) (Fig. 1). El consumo de antiasmáticos en lactantes y preescolares fue mayor en otoño y en invierno, y disminuyó a la mitad en primavera. En escolares y adolescentes predominó en primavera.

La prescripción de antiasmáticos fue más elevada en los niños más pequeños, fundamentalmente en los menores de cuatro años. El pico máximo de consumo de broncodilatadores se produjo en los dos primeros años de vida, siendo su uso muy inferior y estable entre los seis y los 14 años de edad. Los broncodilatadores inhalados predominaron a todas las edades
(6 PDHD inhalados/1,5 PDHD orales) pero los sistémicos tuvieron el consumo más intenso en los menores de dos años, a quienes se prescribió el $45,8 \%$ de los envases de salbutamol oral (prescripción al margen de su autorización en ficha técnica) (Fig. 1). La utilización de LABA fue anecdótica a edades inferiores a las autorizadas (11 envases de formoterol en menores de cinco años y 41 de salmeterol en menores de cuatro años).

En el tratamiento de mantenimiento, los fármacos más prescritos a todas las edades fueron los ALT (casi exclusivamente montelukast), excepto en los

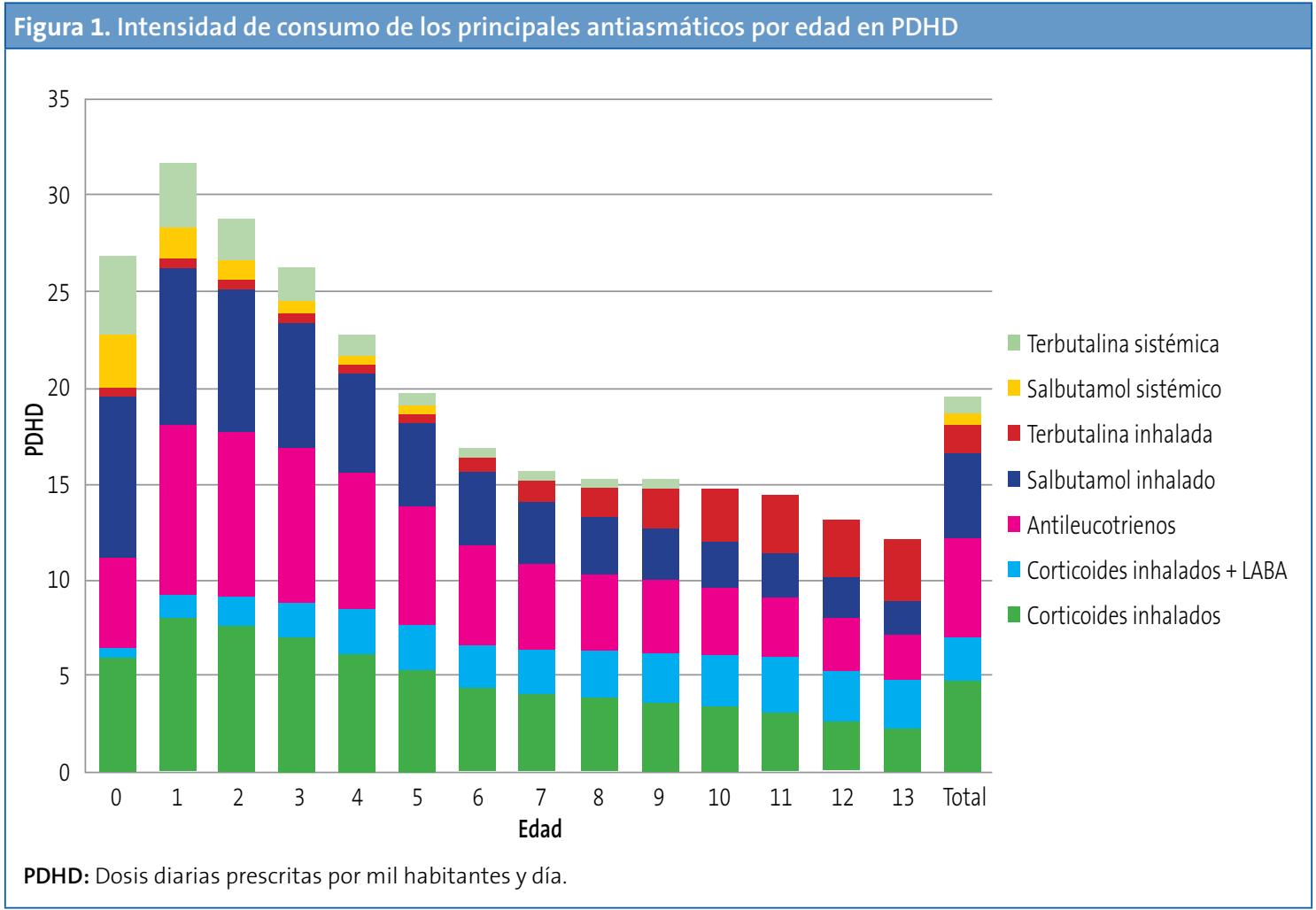


menores de un año, en que fueron superados por los $\mathrm{Cl}$, y a los 13 años en que predominaron los LABA-CI. A la edad de un año se produjo el máximo consumo de ALT (8,9 PDHD) y de Cl (7,9 PDH); y a los 11 años el de LABA-Cl (2,9 PDHD) (Fig. 1).

Respecto a la prescripción al margen de autorización por edad y/o dosis, ocurrió con el 15,9\% de las prescripciones de la terapia de mantenimiento. En la Tabla 3 se exponen los resultados de la prescripción al margen de la ficha técnica: los $L A B A-C I$ se prescribieron desde los primeros meses de vida, aumentando su uso con la edad, pero consumiendo los menores de cuatro años el $14,8 \%$ de las prescripciones totales. El $12 \%$ de las prescripciones de montelukast, el $18,2 \%$ de las de budesonida y el $10,5 \%$ de las de propionato de fluticasona se consumieron a edades no autorizadas. En cuanto al uso de dosis muy elevadas de $\mathrm{Cl}$, sucedió con el 3,9\% de las prescripciones de $\mathrm{Cl}$ y con el $13,1 \%$ de las de $\mathrm{LABA}-\mathrm{Cl}$. El 35\% de prescripciones de salmeterol-fluticasona se recetaron a dosis $\mathrm{y} / \mathrm{o}$ edades al margen de ficha técnica.

\section{DISCUSIÓN}

Este trabajo ha pretendido conocer la intensidad de prescripción de antiasmáticos en niños en condiciones no autorizadas en su ficha técnica en tres aspectos: la indicación, la edad y la dosis. En él se muestra que el consumo de estos medicamentos en niños menores de cuatro años en Castilla y León ha sido muy elevado, especialmente en menores de dos años, siendo probable que en gran proporción se hayan utilizado para tratar entidades con síntomas parecidos a los del asma en los que su indicación es controvertida o escasa. El estudio también expone un uso importante de salbutamol sistémico en menores de dos años, edad no aprobada en su ficha técnica, y un empleo notable de prescripciones de la terapia de mantenimiento al margen de su autorización por edad y/o por uso a dosis superiores a las recomendadas.

En este estudio, existen indicios de que hubo prescripción off-label por indicación no autorizada, ya que se objetiva un elevado consumo de medicación antiasmática en niños pequeños. La estacionalidad

Tabla 3. Prescripciones off-label de antiasmáticos por edad y/o dosis en Castilla y León

\begin{tabular}{|c|c|c|c|c|}
\hline Código ATC & Principio activo & $\begin{array}{l}\text { Número total } \\
\text { prescripciones }\end{array}$ & $\begin{array}{c}\text { Número de prescripciones } \\
\text { off-label }\end{array}$ & $\begin{array}{c}\% \text { de prescripciones } \\
\text { off-label }\end{array}$ \\
\hline $\mathrm{RO} 3 \mathrm{CCO} 2$ & Salbutamol sistémico & 28531 & $13078^{1}$ & $45,8^{1}$ \\
\hline RO3AC12 & Salmeterol & 271 & $41^{1}$ & $15,1^{1}$ \\
\hline RO3AC13 & Formoterol & 549 & $11^{1}$ & $2^{1}$ \\
\hline \multicolumn{2}{|c|}{ Total broncodilatadores } & 189088 & 13124 & $6,9 \%^{1}$ \\
\hline RO3BA02 & Budesonida inhalada & 25968 & $4731^{1}$ & $18,2^{1}$ \\
\hline RO3BA05 & Fluticasona inhalada & 35111 & $\begin{array}{c}2754^{2} \\
3528^{1} \\
154^{4}\end{array}$ & $\begin{array}{c}8,3^{2} \\
10,5^{1} \\
0,4^{4}\end{array}$ \\
\hline \multicolumn{2}{|c|}{ Total corticoides inhalados monofármaco } & 73456 & 11167 & $15,2 \%$ \\
\hline RO3AK07 & Formoterol en asociación & 5840 & $188^{1}$ & $3,2 \%^{1}$ \\
\hline RO3AK06 & Salmeterol en asociación & 19307 & $\begin{array}{c}3210^{2} \\
3473^{1} \\
93^{4}\end{array}$ & $\begin{array}{c}17,1 \%^{2} \\
17,1^{1} \\
0,5^{4}\end{array}$ \\
\hline \multicolumn{2}{|l|}{ Total LABA-Cl } & 25213 & 6964 & $27,6 \%$ \\
\hline RO3DC03 & Montelukast & 106706 & $12964^{3}$ & $12,1^{3}$ \\
\hline \multicolumn{2}{|c|}{ Total terapia mantenimiento } & 195778 & 31095 & $15,9 \%$ \\
\hline
\end{tabular}

${ }^{1}$ Porcentaje de prescripciones off-label por edad no indicada.

${ }^{2}$ Porcentaje de prescripciones off-label por dosis altas de corticoides inhalados.

${ }^{3}$ Porcentaje de prescripciones off-label por dosis (altas o bajas) de montelukast.

${ }^{4}$ Porcentaje de prescripciones off-label por dosis altas de corticoides inhalados y edad no indicada.

LABA-CI: corticoides inhalados asociados a $\beta 2$-adrenérgicos de acción larga. 
en el uso de los antiasmáticos también apoya esta hipótesis, dado que el consumo en lactantes y prescolares fue mayor en otoño-invierno, periodo en el que son más frecuentes las infecciones respiratorias y disminuyó a la mitad en primavera-verano ${ }^{37}$. En primavera es cuando predominan los pólenes de gramíneas, que son los alérgenos más frecuentes en el asma infantil en nuestra comunidad autónoma. Por todo ello, aunque no disponemos de información de diagnósticos clínicos, parece poco probable que la indicación principal en el uso de antiasmáticos en los niños de menor edad haya sido el asma.

El empleo de antiasmáticos en el tratamiento de niños pequeños y su uso off-label es algo muy documentado en la literatura médica $4,6,12,14,17,18,38-40$, siendo el salbutamol $\left.\right|^{4,6,12,17,18,40}$ y los $\mathrm{Cl}^{4,14,17}$, los fármacos más frecuentemente implicados. En algunas de estas investigaciones también se demuestra ${ }^{39} 0$ se interpreta ${ }^{6,18,38}$ que los antiasmáticos se utilizaron para otras indicaciones diferentes al asma.

Pero aparte de la utilización de medicamentos en indicaciones no autorizadas, la prescripción off-label predominante en niños es la asociada al uso de fármacos a edades no autorizadas ${ }^{9,}{ }^{14}$, seguida del

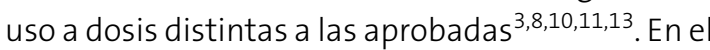
caso de las prescripciones del aparato respiratorio es más frecuente el uso off-label por edad ${ }^{8}$. Con respecto a la edad, en Castilla y León destaca un consumo elevado de broncodilatadores sistémicos y de budesonida inhalada en menores de dos años, y un uso notable de LABA-CI en menores de cuatro años. De los broncodilatadores sistémicos, es reseñable la utilización de salbutamol sistémico en menores de dos años, que no está aprobado en ficha técnica y que tampoco se recomienda en ninguna guía de asma por vía oral, ya que tiene más efectos adversos y un inicio de acción más tardío que por vía inhalada. No es muy común la referencia a este tipo de vía de administración de salbutamol, aunque en EE. UU. (2004-2005) ${ }^{41}$ igualmente hubo un consumo frecuente en niños sin diagnóstico de asma.

En lo que concierne a los LABA-CI, según este estudio (especialmente salmeterol asociado a fluticasona), se prescribieron en pequeña proporción en los menores de un año, pero entre los 2-3 años tuvieron un nivel similar de consumo que de los siete a los diez años. Esto también se ha observado en Andalucía ${ }^{42}$ en menores de cuatro años. El consumo en niños de LABA asociados o no a los $\mathrm{Cl}$ es un asunto controvertido por los riesgos que parece conllevar de efectos adversos. Diversas investigaciones han encontrado mayor riesgo de efectos adversos graves, especialmente hospitalizaciones, en niños usuarios de LABA asociados o no a $\mathrm{Cl}^{43-45}$. Y este riesgo es desconocido o muy poco estudiado en menores de cuatro años. Es preocupante por tanto que en nuestro estudio haya habido un consumo importante a estas edades.

Por otro lado, y en referencia a la prescripción offlabel de antiasmáticos por la dosis utilizada, el $4 \%$ de las prescripciones de $\mathrm{CI}$ monofármaco y el $13 \%$ de las de los LABA-CI se utilizaron a dosis muy altas. Especialmente con fluticasona y en niños mayores de cuatro años. Situaciones similares se describen en otros estudios. Así, en Reino Unido (1999-2000) los CI monofármaco fueron los antiasmáticos más implicados en el uso off-label a altas dosis a todas las edades pero especialmente en mayores de cinco años ${ }^{10}$, lo que se atribuye a la discordancia entre las dosis aconsejadas en las fichas técnicas y en la guía británica del asma. En Holanda (2002) ${ }^{46}$ un $8 \%$ de los niños que inician $\mathrm{Cl}$ lo hacen con dosis mucho más elevadas de las aconsejadas (el doble), fundamentalmente con fluticasona y en mayores de diez años. Los autores desconocen por qué se utilizaron dosis tan elevadas, aunque que creen que podría deberse a que en Holanda hay presentaciones de 100, 250 y $500 \mu \mathrm{g}$. Presentaciones que también tenemos en España además de la de $50 \mu \mathrm{g}$. En Portugal (2012) se ha publicado una utilización off-label por dosis altas para la edad, de casi la mitad de las prescripciones de fluticasona en preescolares ${ }^{7}$. El consumo de $\mathrm{Cl}$ de forma prolongada a dosis altas o muy altas tiene como riesgos la disminución en la talla adulta final ${ }^{47}$ y la posible supresión del eje hipotálamo-hipófisis-suprarrenal, especialmente en niños con dosis iguales o mayores a 500 Mg/día de fluticasona inhalada o equivalente ${ }^{48}$. Riesgos 
que habría que tener en cuenta, ya que la mayoría de los niños con asma persistente están bien controlados con dosis bajas-medias de $\mathrm{Cl}$, con las que el crecimiento lineal parece no estar afectado ${ }^{49}$. Solo en aquellos con asma de difícil control (alrededor del 2\% de niños asmáticos en España ${ }^{50}$ ), estarían indicadas dosis mayores de $800 \mu \mathrm{g} /$ día de budesonida o equivalente.

En cuanto a montelukast, en Castilla y León un 12\% de las prescripciones se prescribieron a dosis para la edad al margen de su ficha técnica, un $8 \%$ del total a dosis más elevadas de las aconsejadas. Montelukast se ha utilizado en los ensayos clínicos ${ }^{51,52}$ a dosis variables en menores de 36 meses (4 y $8 \mathrm{mg}$ ) y se ha comparado con placebo, sin encontrar diferencias entre los tres grupos en la aparición de efectos adversos. Pero los estudios poscomercialización han detectado eventos neuropsiquiátricos a todas las edades, motivo por el que en 2009 la FDA ${ }^{53}$ emitió una alerta de seguridad con recomendaciones para los profesionales de la salud y en 2011 la AEMPS $^{54}$ la incluyó también. Así mismo, en Suecia, el estudio de las reacciones adversas psiquiátricas entre 2001-2010 en los menores de 18 años detectó que fueron más frecuentes y graves cuando los fármacos se utilizaron al margen de ficha técnica y entre las drogas más habitualmente implicadas estaban montelukast y budesonida inhalada ${ }^{55}$ : con montelukast predominaron los trastornos del sueño y con los CI la agresividad. El uso a dosis altas y la corta edad fueron las situaciones más habituales de uso off-label con estos fármacos.

Los antiasmáticos son unos de los grupos de medicamentos pediátricos en los que se refiere mayor número de reacciones adversas y estas son más frecuentes cuando se utilizan off-labe ${ }^{28,30}$. En Suecia $(2000)^{30}$ los antiasmáticos fueron el grupo terapéutico con el que se sospecharon más reacciones adversas en niños no hospitalizados, y la tercera parte de ellas asociadas a un uso off-label. Si tenemos en cuenta que la notificación de efectos adversos de medicamentos es escasa y, como señalan otros estudios, es posible que muchos pediatras no hayan sido conscientes de haber realizado una prescripción off-label ${ }^{9,16,56}$, parece probable que el número de reacciones adversas acontecidas sea mucho mayor.

Finalmente nos gustaría comentar la limitación principal de este trabajo, ya que solo permite conocer una parte de la prescripción de antiasmáticos al margen de su autorización debido a que la base de datos farmacéutica utilizada no tiene conexión con historias clínicas de los pacientes. Así, se ignoran los diagnósticos para los que se prescribieron y se han estimado las dosis en función del tipo de formato utilizado a cada edad y de la pauta posológica aconsejada en ficha técnica. Tampoco se conocen la gravedad del asma, ni el tiempo de consumo de estos medicamentos. No obstante, la fortaleza del estudio es su base poblacional y su tamaño. Así, las prescripciones analizadas probablemente representan el $65 \%$ de los antiasmáticos consumidos por los menores de 14 años de Castilla y León en este periodo ${ }^{37}$ y ello permite valorar la realidad de este tipo de prescripción en nuestra comunidad.

En conclusión, este estudio muestra una parte de la prescripción pediátrica de antiasmáticos realizada al margen de su autorización en ficha técnica en la comunidad autónoma de Castilla y León, en un periodo reciente. Señala que la mayor intensidad de consumo se ha realizado a edades en las que es menos probable el diagnóstico de asma, y objetiva un uso a edades y con dosis en el que el riesgo-beneficio puede ser desfavorable. Aunque la prescripción sin autorización puede ser necesaria y adecuada, debiera apoyarse en criterios científicos sólidos y buscar un beneficio para el paciente.

\section{CONFLICTO DE INTERESES}

Los autores declaran no presentar conflictos de intereses en relación con la preparación y publicación de este artículo.

\section{ABREVIATURAS}

AEMPS: Agencia Española de Medicamentos y Productos Sanitarios • ALT: inhibidores de los leucotrienos • AP: Atención Primaria - ATC: Anatomical Therapeutic Chemical Classification $\bullet \mathrm{Cl}$ : corticoides inhalados • CONCYLIA: Sistema de Información de Farmacia de Castilla y León - EMA: European Medicines Agency $\bullet$ FDA: Food and Drug Administration • PDD: dosis diaria prescrita $\bullet$ PDHD: dosis diaria prescrita por mil habitantes y día - SACYL: Sistema Nacional de Salud de Castilla y León • LABA: broncodilatadores de acción larga. 


\section{BIBLIOGRAFİA}

1. Neubert A, Wong IC, Bonifazi A, Catapano M, Felisi M, Baiardi $P$, et al. Defining off-label and unlicensed use of medicines for children: Results of a Delphi survey. Pharmacol Res. 2008;58:316-22.

2. Turner S, Longworth A, Nunn AJ, Choonara I. Unlicensed and off label drug use in paediatric wards: Prospective study. BMJ. 1998;316:343-5.

3. Pandolfini Ch, Bonati M. A literature review on off-label drug use in children. Eur J Pediatr. 2005;164:552-8.

4. Conroy S, Choonara I, Impicciatore P, Mohn A, Arnell $H$, Rane $A$, et al. Survey of unlicensed an off label drug use in paediatric wards in European countries. BMJ. 2000;320:79-83.

5. Lindell-Osuagwu L, Korhonen MJ, Saano S, HelinTanninen M, Naaranlahti T, Kokki H. Off-label and unlicensed drug prescribing in three paediatrics wards in Finland and review of the international literature. J Clin Pharm Ther. 2009;34:277-87.

6. Baiardi P, Ceci A, Felisi M, Cantarutti L, Girotto S, Sturkenboom $M$, et al. In-label and off-label use of respiratory drugs in the Italian paediatric population. Acta Paediatr. 2010;99:544-9.

7. Morais-Almeida M, Cabral AJ. Off-label prescribing for allergic diseases in pre-school children. Allergol Immunopathol (Madrid). 2014;42:342-7.

8. Silva D, Ansotegui I, Morais-Almeida M. Off-label prescribing for allergic diseases in children. World Allergy Ongan J. 2014;7:4.

9. Chalumeau M, Treluyer JM, Salanave B, Assathiany R, Cheron $\mathrm{G}$, Crocheton N, et al. Off-label and unlicensed drug use among French office based paediatricians. Arch Dis Child. 2000;83:502-5.

10. Ekins-Daukes S, Helms PJ, Simpson CR, Taylor MW, McLay JS. Off-label prescribing to children in primary care: retrospective observational study. Eur J Clin Pharmacol. 2004;60:349-53.

11. Gavrilov V, Lifshitz M, Levy J, Gorodischer R. Unlicensed and off-label medication use in a General Pediatrics Ambulatory Hospital unit in Israel. IMAJ. 2000;2:595-7.

12. Bazzano AT, Mangione-Smith R, Schonlau M, Suttorp MJ, Brook RH. Off-label prescribing to children in the United States outpatient setting. Acad Pediatr. 2009; 9:81-8.
13. Morales-Carpi C, Estañ L, Rubio E, Lurbe E, MoralesOlivas FJ. Drug utilization and off-label drug use among Spanish emergency room paediatric patients. Eur J Clin Pharmacol. 2010;66:315-20.

14. Blanco-Reina E, Vega-Jiménez MA, Ocaña-Riola M, Márquez Romero El, Bellido-Estévez I. Estudio de las prescripciones farmacológicas en niños a nivel de atención primaria: evaluación de los usos off-label o fuera de ficha técnica. Aten Primaria. 2014;47:344-50.

15. Garcia Blanes CP, Rodríguez-Cantón Pascual P, Morales-Carpi C, Morales-Olivas FJ. ¿Se ha modificado el uso de antitérmicos tras la introducción de ibuprofeno a diferentes concentraciones? An Pediatr (Barc). 2014;81:383-8.

16. Piñeiro Pérez R, Ruiz Antorán MB, Avendaño Solá $C$, Román Riechmann E, Cabrera García L, Cilleruelo Ortega MJ, et al. Conocimiento sobre el uso de fármacos off-label en Pediatría. Resultados de una encuesta pediátrica nacional 2012-2013 (estudio OLPED). An Pediatr (Barc). 2014;81:16-21.

17. t’Jong GW, Eland IA, Sturkenboom MC, van den Anker $\mathrm{JN}$, Strickerf BH. Unlicensed and off-label prescription of respiratory drugs to children. Eur Respir J. 2004;7:310-3.

18. Sen EF, Verhamme KM, Neubert A, Hsia Y, Murray M, Felisi $M$, et al. Assessment of pediatric asthma drug use in three European countries; a TEDDY study. Eur J Pediatr. 2011;170:81-92.

19. McCowan C, Hoskins G, Neville RG. Clinical symptoms and 'off-label' prescribing in children with asthma. Br J Gen Pract. 2007;57:220-2.

20. Wharton GT, Murphy MD, Avant D, Goldsmith JV, Chai G, Rodríguez VJ, et al. Impact of pediatric Exclusivity on drug labeling and demonstrations of efficacy. Pediatrics. 2014;134:e512-e518.

21. American Academy of Pediatrics. Committe on Drugs. Off-label use drugs in children. Pediatrics. 2014;133:563-7.

22. Informe de la comisión al parlamento europeo y al consejo. Mejores medicamentos pediátricos. Del concepto a la realidad. En: Comisión Europea [en línea] [consultado el 27/08/2015. Disponible en http://ec.europa.eu/health/files/paediatrics/2013_ com443/paediatric_report-com(2013)443_es.pdf

23. Peiré García MA. Importancia de la farmacología clínica en pediatría. An Pediatr (Barc). 2010;72:99-102. 
24. Valls-i-Soler A, Santesteban E, Campino A. Mejores medicamentos en Pediatría. An Pediatr (Barc). 2011; 75:85-8.

25. Bravo Acuña J. Mesa Redonda. Uso de fármacos offlabel en las diferentes disciplinas pediátricas. Libro de ponencias y comunicaciones. Congreso extraordinario de la Asociación Española de Pediatría 2014 (p. 184). En: Congresoaep.org [en línea] [consultado el 27/08/2015]. Disponible en www.congresoaep. org/2014/readcontents. php?file=webstructure $/ 01$ sesiones_cientificas_oficiales.pdf

26. Sachs AN, Avant D, Lee CS, Rodriguez W, Murphy MD. Pediatric information in drug product labeling. JAMA. 2012;307:1914-5.

27. Real Decreto 1015/2009 por el que se regula la disponibilidad de medicamentos en situaciones especiales. Boletín Oficial del Estado número 174 de lunes 20 de julio del 2009. Disponible en www.boe.es/ boe/dias/2009/07/20/pdfs/BOE-A-2009-12002.pdf

28. Turner S, Nunn AJ, Fielding K, Choonara I. Adverse drug reactions to unlicensed and off-label drugs on paediatric wards: a prospective study. Acta Paediatr. 1999;88:965-8.

29. Evidence of harm from off-label or unlicensed medicines in children; EMA/11207/04. En: European Mecicines Agency [en línea] [consultado el 27/08/2015]. Disponible en www.ema.europa.eu/ docs/en_GB/document_library/Other/ 2009/10/ WC500004021.pdf

30. Ufer M, Kimland, E, Bergman U. Adverse drug reactions and off-label prescribing for paediatric outpatients: a one-year survey of spontaneous reports in Sweden. Pharmacoepidemiol Drug Saf. 2004;13:147-52.

31. Mason J, Pirmohamed M, Nunn T. Off-label and unlicensed medicine use and adverse drug reactions in children: a narrative review of the literature. Eur J Clin Pharmacol. 2012;68:21-8.

32. Horen B, Montastruc JL, Lapeyre-Mestre M. Adverse drug reactions and off-label drug use in paediatric outpatients. Br J Clin Pharmacol. 2002;54:665-70.

33. National Asthma Education and Prevention Program. Expert Panel Report 3: Guidelines for the diagnosis and management of asthma. Full report 2007. En: National Heart, Lung, and Blood Institute [en línea] [consultado el 27/08/2015]. Disponible en www. nhlbi.nih.gov/guidelines/asthma/asthgdln.pdf
34. Global Strategy for Asthma Management and Prevention. Revised 2014. En: Global Initiative for Asthma [en línea] [consultado el 27/08/2015, actualizado en 2014]. Disponible en www.ginasthma.org

35. Castillo JA, de Benito J, Escribano A, Fernández M, García de la Rubia S, Grupo de trabajo para el Consenso sobre Tratamiento del Asma Infantil. Consenso para el tratamiento del asma en Pediatría. An Pediatr (Barc). 2007;67:253-73.

36. British guideline on the management of asthma. En: SIGN [en línea] [consultado el 27/08/2015, actualizado en octubre de 2014]. Disponible en www.sign. ac.uk/pdf/SIGN141.pdf

37. Casares-Alonso I, Cano-Garcinuño A, Blanco-Quirós A, Pérez-García A. Anti-asthmatic prescription variability in children according to age. Allergol Immunopathol (Madr). 2015;43:383-91.

38. Bisgaard H, Szfler S. Prevalence of asthma-like symptoms in young children. Pediatr Pulmonol. 2007; 42:723-38.

39. Ochoa Sangrador C, González de Dios J, Grupo investigador del proyecto aBREVIADo (BRonquiolitisEstudio de Variabilidad, Idoneidad y ADecuación). Manejo de la bronquiolitis aguda en atención primaria: análisis de la variabilidad e idoneidad (proyecto aBREVIADo). An Pediatr (Barc). 2013;79:167-76.

40. Ribeiro M, Jorge A, Macedo AF. Off-label Drug prescribing in a Portuguese paediatric emergency unit. Int J Clin Pham. 2013;35:30-6.

41. Korelitz JJ, Zito JM, Gavin NI, Masters MN, McNally D, Irwin DE, et al. Asthma-related medication use among children in the United States. Ann Allergy Asthma Immunol. 2008;100:222-9.

42. Praena Crespo M, Lora Espinosa A, Murcia Garcia J, Rodriguez Castilla J. Uso racional de medicamentos en el asma y en el menor de tres años con sibilancias. En: AEPap. Curso de Actualizacion en Pediatría 2011. Madrid: Exlibris Ediciones; 2011. p. 391-4.

43. McMahon AW, Levenson MS, McEvoy BW, Mosholder $A D$, Murphy, D. Age and risks of FDA-approved longacting $\beta 2$-adrenergic receptor agonists. Pediatrics. 2011;128:e1147-e1154.

44. Rodrigo GJ, Castro-Rodríguez JA. Safety of long-acting beta agonists for the treatment of asthma: clearing the air. Thorax. 2012;67:342-9.

45. Cates CJ, Cates MJ. Regular treatment with formoterol for chronic asthma: serious adverse events. 
Cochrane Database Syst Rev. 2012 Apr 18;4: CD006923.

46. Schirm E, de Vries TW, Tobi $H$, van den Berg PB, de Jong-van den Berg LT. Prescribed doses of inhaled steroids in Dutch children: too little or too much, for too short a time. Br J Clin Pharmacol. 2006;62:383-90.

47. Pruteanu Al, Chauhan BF, Zhang L, Prietsch SO, Ducharme FM. Inhaled corticosteroids in children with persistent asthma: is there a dose response impact on growth? An overview of Cochrane reviews. Paediatr Respir Rev. 2015;16:51-2.

48. Smith RW, Downey K, Gordon M, Hudak A, Meeder R, Barker $S$, et al. Prevalence of hypothalamic-pituitaryadrenal axis suppression in children treated for asthma with inhaled corticosteroid. Paediatr Child Health. 2012;17:e34-e39.

49. Smyth AR, Barbato A, Beydon N, Bisgaard H, de Boeck $\mathrm{K}$, Brand $\mathrm{P}$, et al. Respiratory medicines for children: current evidence, unlicensed use and research priorities. Eur Respir J. 2010;35:247-65.

50. Plaza-Martín AM, Vennera MC, Galera J, Herráez L, PREX Study Group. Prevalence and clinical profile of difficult-to-control severe asthma in children: Results from pneumology and allergy hospital units in Spain. Allergol Immunopathol (Madr). 2014;42: 510-7.

51. Bisgaard H, Study Group on Montelukast and Respiratory Syncitial Virus. A randomized trial of montelukast in respiratoty syncytial virus postbronchiolitis. Am J Respir Crit Care Med. 2003;167:379-83.
52. Bisgaard H, Flores-Nunez A, Goh A, Azimi P, Halkas A, Malice MP, et al. Study of montelukast for the treatment or respiratory symptoms of post-respiratory syncitial virus bronchiolitis in children. Am J Respir Crit Care Med. 2008;178:854-60.

53. Leukotriene Inhibitors: Montelukast (marketed as Singulair), Zafirlukast (marketed as Accolate), and Zileuton (marketed as Zyflo and Zyflo CR). 2009. En: FDA. Safety information [en línea] [consultado el 27/08/2015]. Disponible en www.fda.gov/Safety/ MedWatch/Safetylnformation/SafetyAlertsforHu manMedicalProducts/ucm166246.htm

54. Información sobre seguridad. Montelukast: notificación de casos de reacciones psiquiátricas. En: AEMPS [en línea] [consultado el 27/08/2015]. Disponible en www.aemps.gob.es/informa/informeMensual/ 2011/febrero/informe-medicamentos.htm

55. Byddell M, Brunlöf G, Wallerstedt SM, Kindblom JM. Psychiatric adverse drug reactions reporting during a 10-year period in the Swedish pediatric population. Pharmacoepidemiol Drug Saf. 2012;2179-86.

56. Marchetti F, Bua J, Ventura A, Notarangelo LD, Di Maio S, MiglioriG, et al. The awareness among paediatricians of off-label prescribing in children: a survey of Italian hospitals. Eur J Clin Pharmacol. 2007; 63:81-5. 